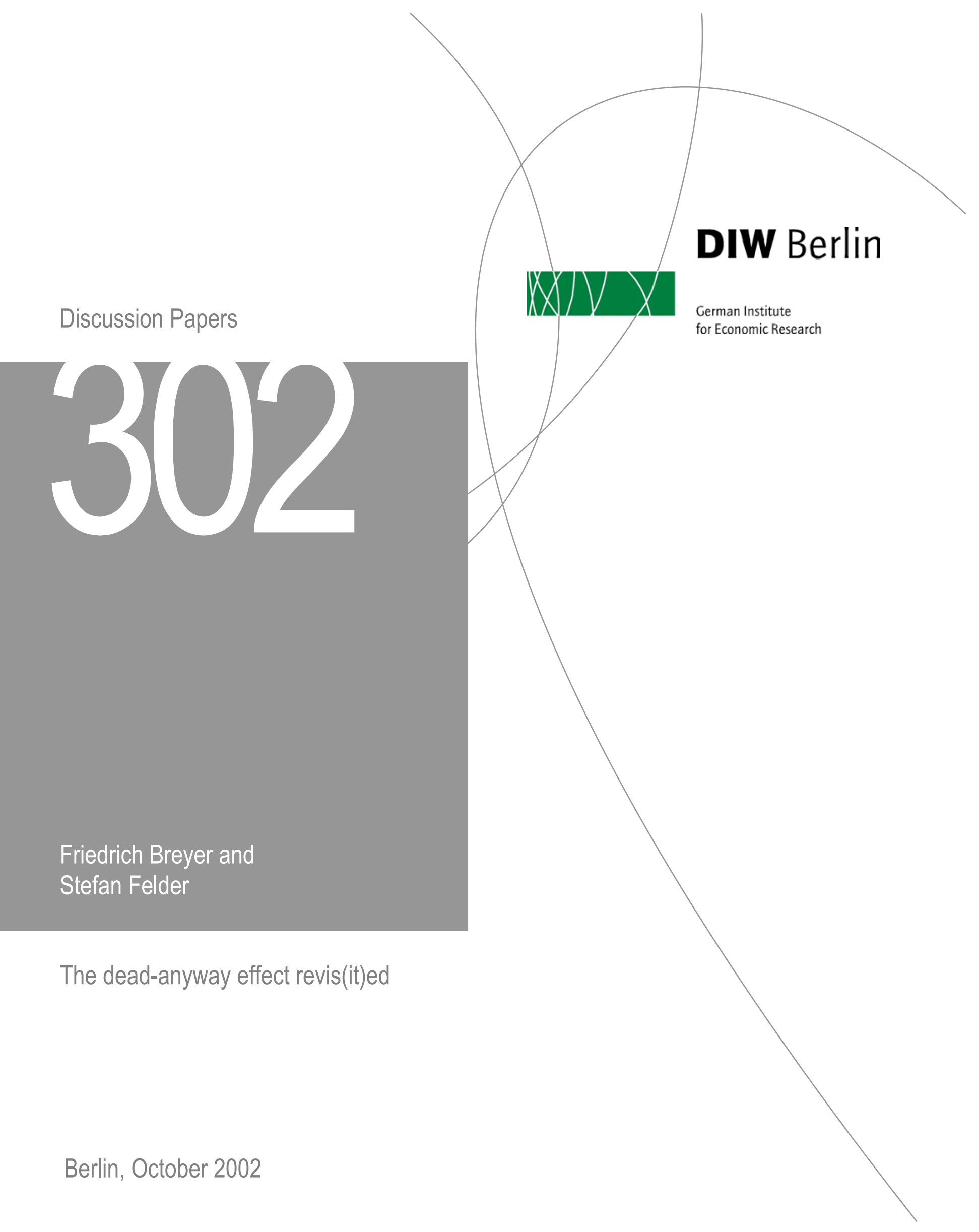


Opinions expressed in this paper are those of the author and do not necessarily reflect views of the Institute.

\section{DIW Berlin}

\section{German Institute}

for Economic Research

Königin-Luise-Str. 5

14195 Berlin,

Germany

Phone +49-30-897 89-0

Fax +49-30-897 89-200

www.diw.de

ISSN 1619-4535 


\title{
The dead-anyway effect revis(it)ed ${ }^{*}$
}

\author{
by \\ Friedrich Breyer, University of Konstanz and DIW Berlin \\ Stefan Felder, University of Magdeburg
}

First version, October 6, 2002

Keywords: Value of life, expected utility, willingness to pay, insurance markets

JEL classification: D8, H43, I18

Abstract: In the expected-utility theory of the monetary value of a statistical life, the so-called "dead-anyway" effect discovered by Pratt and Zeckhauser (1996) asserts that an individuals' willingness to pay (WTP) for small reductions in mortality risk increases with the initial level of risk. Their reasoning is based on differences in the marginal utility of wealth between the two states of nature: life and death. However, this explanation is based on the absence of markets for contingent claims, i.e. annuities and life insurance. This paper reexamines the "dead-anyway" effect and establishes two main results: first, for a risk-averse individual without a bequest motive, marginal WTP for survival does increase with the level of risk but when insurance markets are perfect, this occurs for a different reason than given by Pratt and Zeckhauser. Secondly, when the individual has a bequest motive and is endowed with a sufficient amount of non-inheritable capital, the effect of initial risk on WTP for survival is reversed: the higher initial risk the lower the value of a statistical life.

\footnotetext{
* The research for this paper began while the first author was a visiting scholar at the Research School of Social Sciences (Social \& Political Theory Group) of the Australian National University, Canberra. Valuable comments by Mathias Kifmann, Normann Lorenz (University of Konstanz) and Andreas Werblow (University of Magdeburg) are gratefully acknowledged.
} 


\section{Introduction}

The expected-utility foundation of the (monetary) value of a statistical life is based on the seminal contributions by Jones-Lee (1976, chapter 5) and Rosen (1988). A key question in this literature is how this value, i.e. the willingness to pay (WTP) for small increments in the chance of survival is influenced by the characteristics of the person under consideration, in particular by his (inheritable or non-inheritable) wealth and the initial value of the mortality risk. This question is of significant policy relevance because in most practical applications, marginal WTP of the exposed persons can not be directly observed but must be inferred from e.g. questionnaire responses of a different population, which is often much less exposed. If there is a systematic relationship between WTP and the level of exposure, then such an inference may be unwarranted if the results are not appropriately corrected.

The current state of knowledge on this question is based on a paper by Pratt and Zeckhauser (1996) who claim that an individual's WTP will be monotonically increasing in the initial level of risk due to a "dead-anyway" effect (henceforth: DAE). This means that if marginal utility of wealth when dead (or marginal utility of bequest) is lower than marginal utility of wealth when alive (or marginal utility of consumption), then increasing the mortality risk reduces the expected marginal utility of wealth and thus increases WTP, which is defined as the marginal rate of substitution between wealth and the chance of survival.

However, this explanation may be simplistic because it is based on the complete absence of contingent-claims markets (i.e. annuities and life insurance) where the individual can trade wealth across different states of nature and thus equalize marginal utility of wealth. It is a priori unclear whether this result will hold in a world of perfect contingent-claims markets. Furthermore, while it seems plausible that WTP increases with the individual's wealth (see, e.g., Jones-Lee 1976, p. 99), it is not so clear whether this is true for all kinds of wealth including non-inheritable like human capital, which is obviously lost upon death.

The present paper is devoted to a thorough theoretical analysis of the determinants of WTP for small increments in the chance of survival under various circumstances. The main question to be answered is whether the "dead-anyway effect" is invariably true or holds only under additional assumptions and whether the intuitive explanation of this effect given by Pratt and Zeckhauser is correct.

The paper is organized as follows. Section 2 introduces the basic model without a bequest motive, Section 3 adds bequests, Section 4 deals with the case of imperfect insurance markets, 
Section 5 combines these two extensions and Section 6 summarizes the findings and compares them to the available empirical evidence:

\section{The basic model: perfect insurance markets and no bequest motive}

We consider an individual whose (indirect) utility $u(W)$ is a positive and strictly increasing function of her wealth $W$ provided she stays alive, i.e. $u>0, u^{\prime}>0$. With zero utility assigned to the state of death, the expected utility of the individuals is:

$$
E U=\pi \cdot u(W)
$$

where $\pi$ is the probability of survival. Total wealth is composed of non-inheritable wealth $H$, which is lost upon death, and inheritable wealth $V$. Non-inheritable wealth includes human capital and annuities that already exist at the time the individual reveals her willingness to pay for a change in survival probability. Assume that there is an insurance market where survivors are offered fair tontine shares. Individuals then will seek to trade their non-human endowment $V$ for a contract which pays $V$ plus the endowment of those who die $[(1-\pi) / \pi] \cdot V$, or $V / \pi$ in all (Rosen, 1988). The individual faces the following budget constraint:

$$
\pi \cdot W=V+\pi \cdot H
$$

The marginal willingness to pay $d V$ for a small increase in survival probability $d \pi$ equals the marginal rate of substitution between survival and inheritable wealth:

$$
m(\pi, V, H)=-\left.\frac{d V}{d \pi}\right|_{d E U=0}=\frac{\frac{\partial E U}{\partial \pi}}{\frac{\partial E U}{\partial V}}=\frac{u-u^{\prime} \cdot V / \pi}{u^{\prime}}=\frac{u}{u^{\prime}}+(H-W) .
$$

$H-W$ is the gradient for the change in expected income from an increase in marginal survival, providing a lower bound for WTP for survival. The assumption of perfect insurance markets is crucial for the derivation of (3). It means that insurers will adjust the tontine if the survival probability changes. Old-age insurance schemes that include a demographic factor for changes in life expectancy, which is used in Scandinavia and was discussed but not implemented in Germany, fulfill this requirement. However, whether this is guaranteed on an individual level is doubtful.

The derivative of $m(\pi, V, H)$ with respect to $\pi$ gives: 


$$
\frac{\partial m(\pi, V, H)}{\partial \pi}=-\left.\frac{\partial^{2} V}{\partial \pi^{2}}\right|_{d E U=0}=\frac{-\left(u^{\prime}\right)^{2} \cdot \frac{V}{\pi^{2}}+u \cdot u^{\prime \prime} \cdot \frac{V}{\pi^{2}}}{\left(u^{\prime}\right)^{2}}-\frac{V}{\pi^{2}}=\frac{V \cdot u^{\prime \prime} \cdot u}{\left(\pi \cdot u^{\prime}\right)^{2}}
$$

It turns out that the DAE asserted by Pratt and Zeckhauser (1996), i.e. a negative sign of $\partial m / \partial \pi$, occurs if and only if $u "<0$, which means that the individual is risk-averse.

The economic intuition behind this result is quite different from the one given by Pratt and Zeckhauser, who claim that a smaller probability of survival decreases the expected utility cost of the funds spent on risk reduction by increasing the chance that the money will be drawn from the zero-utility state of death. The explanation which fits this perfect-markets case is that decreasing the survival chance $\pi$ raises the tontine share in the state of survival, $V / \pi$, and thus with a concave utility function lowers the marginal utility of wealth, which in turn means that more wealth will be sacrificed for a given increase in the probability of survival.

For the derivative with respect to non-inheritable wealth $V$ we obtain from (3), using (2):

$$
\frac{\partial m(\pi, V, H)}{\partial V}=\frac{\left(u^{\prime}\right)^{2} \cdot \frac{1}{\pi}-u \cdot u^{\prime \prime} \cdot \frac{1}{\pi}}{\left(u^{\prime}\right)^{2}}-\frac{1}{\pi}=-\frac{u \cdot u^{\prime \prime}}{\pi \cdot\left(u^{\prime}\right)^{2}} .
$$

Again, with a concave utility function this expression is positive. Thus, survival is a noninferior good (Jones-Lee, 1976) and the economic explanation is the same as above: increasing inheritable wealth has the same effect on the tontine share as raising the risk of death.

We finally differentiate $m$ with respect to non-inheritable wealth $H$ and obtain:

$$
\frac{\partial m(\pi, V, H)}{\partial H}=\frac{\left(u^{\prime}\right)^{2}-u \cdot u^{\prime \prime}}{\left(u^{\prime}\right)^{2}}=1-\frac{u \cdot u^{\prime \prime}}{\left(u^{\prime}\right)^{2}} .
$$

Notice that risk aversion is a sufficient but not a necessary condition for an increase in the value of life with non-inheritable wealth. An increase in non-inheritable wealth has two effects on WTP for survival: first, it raises the part of total wealth that is lost upon death so that the individual is prepared to give up that part to avoid death. This is reflected in the first term on the RHS of (6). Secondly, it raises total wealth and thereby, with concave utility, decreases the marginal utility of wealth. 
This one-period analysis can be extended to a continuous consumption life-cycle model with actuarially fair annuities. Felder et al. (2000) prove for a permanent change of the mortality risk in the sense of Johansson (1996) the DAE to hold.

\section{Adding bequests}

We extend the model by assuming that individuals care for their heirs. Expected utility is defined as follows:

$$
E U=\pi \cdot u_{L}\left(W_{L}\right)+(1-\pi) \cdot u_{D}\left(W_{D}\right)
$$

where $u_{L}$ is utility in the state of survival $(L)$ and $u_{D}$ represents the bequest motive, i.e. utility of the heirs in the state of death $(D)$. The state dependent utilities are both assumed to be strictly increasing: $u_{L}{ }^{\prime}, u_{D}{ }^{\prime}>0$.

As common in the pertinent literature (e.g. Jones-Lee, 1976), we assume that at all values of $W$ individuals prefer survival to death, and that at any level of consumption marginal utility when alive is higher than marginal utility when dead:

$$
\begin{aligned}
& u_{L}(W)>u_{D}(W) \quad \forall W, \\
& u_{L}{ }^{\prime}(W)>u_{D}{ }^{\prime}(W) \quad \forall W .
\end{aligned}
$$

An insurance market offers $1 / \pi$ for any dollar spent on an annuity contract and $1 /(1-\pi)$ for any dollar spent on life insurance. This gives rise to the budget constraint:

$$
\pi \cdot W_{L}+(1-\pi) \cdot W_{D}=V+\pi \cdot H
$$

Maximizing (7) subject to (10) leads to the following FOC for an interior optimum:

$$
u_{L}{ }^{\prime}\left(W_{L}\right)=u_{D}{ }^{\prime}\left(W_{D}\right) \text {. }
$$

This solution is a maximum if the indifference curves for expected utility are convex. In the appendix we show that this requires $a:=\pi \cdot u_{D} "+(1-\pi) \cdot u_{L} "<0$. Concave utility functions in both states of nature $\left(u_{L} " \cdot u_{D} ">0\right)$ satisfy this condition. However, $a<0$ also holds with increasing marginal utility in one state, provided that marginal utility sufficiently decreases in the other state. Convex indifference curves together with (9) imply that $W_{L}-W_{D}>0$ in the optimum. Furthermore, by (8) and the monotonicity of the utility function, utility in the state of survival exceeds utility in the state of death,

$$
u_{L}\left(W_{L}\right)>u_{D}\left(W_{D}\right)
$$


To derive the marginal rate of substitution between survival and wealth, we totally differentiate expected utility and set the differential equal to zero. Expressing $W_{L}\left(W_{D}\right)$ using the budget constraint (10) and the FOC (11), we find after some modifications: ${ }^{1}$

$$
m_{b}(\pi, V, H)=-\left.\frac{d V}{d \pi}\right|_{d E U=0}=\frac{u_{L}-u_{D}}{u_{L}^{\prime}}+\left[H-\left(W_{L}-W_{D}\right)\right] .
$$

Note the similarity between (13) and (3). When bequests are added to the pure consumption model, the value of a statistical life is driven by the utility difference in monetary terms and the gradient for the change in expected wealth.

Since

$$
\begin{aligned}
\frac{\partial\left(\frac{u_{L}-u_{D}}{u_{L}{ }^{\prime}}\right)}{\partial \pi} & =\frac{\left(u_{L}{ }^{\prime} \cdot \frac{\partial W_{L}}{\partial \pi}-u_{D}{ }^{\prime} \cdot \frac{\partial W_{D}}{\partial \pi}\right) \cdot u_{L}{ }^{\prime}-u_{L}{ }^{\prime} \cdot \frac{\partial W_{L}}{\partial \pi} \cdot\left(u_{L}-u_{D}\right)}{\left(u_{L}^{\prime}\right)^{2}} \\
& =\frac{\partial W_{L}}{\partial \pi}-\frac{\partial W_{D}}{\partial \pi}-\frac{\left(u_{L}-u_{D}\right) \cdot u_{L}{ }^{\prime \prime}}{\left(u_{L}{ }^{\prime}\right)^{2}} \cdot \frac{\partial W_{L}}{\partial \pi},
\end{aligned}
$$

we obtain for the derivative of $m$ with respect to $\pi$ at an iso-expected-utility locus in the $\left(W_{L}, W_{D}, \pi\right)$-space from (13):

$$
\frac{\partial m_{b}(\pi, V, H)}{\partial \pi}=-\frac{\partial W_{L}}{\partial \pi} \cdot \frac{\left(u_{L}-u_{D}\right) \cdot u_{L}^{\prime \prime}}{\left(u_{L}^{\prime}\right)^{2}}
$$

In the appendix we show that:

$$
\frac{\partial W_{L}}{\partial \pi}=\left[H-\left(W_{L}-W_{D}\right)\right] \cdot \frac{u_{D}^{\prime \prime}}{a} .
$$

(15) implies that an income effect is only involved when $\pi$ changes in the perfect insurance model. In fact, according to (11) the price of the annuity is not part of the FOC. Hence with constant welfare we do not expect a change in demand for annuities i.e. the compensating price change is zero.

A combination of (14) and (15) leads to:

$$
\frac{\partial m_{b}(\pi, V, H)}{\partial \pi}=-\frac{\left(u_{L}-u_{D}\right)}{\left(u_{L}{ }^{\prime}\right)^{2}} \cdot \frac{u_{L} " \cdot u_{D} "}{a} \cdot\left[H-\left(W_{L}-W_{D}\right)\right], \text { and since } a<0 \text { : }
$$

\footnotetext{
${ }^{1}$ The subscript $b$ at the marginal WTP refers to the case of bequest.
} 


$$
\operatorname{sign}\left(\partial m_{b} / \partial \pi\right)=\operatorname{sign}\left(u_{L} " \cdot u_{D} "\right) \cdot \operatorname{sign}\left[H-\left(W_{L}-W_{D}\right)\right] .
$$

The DAE holds if $u_{L} " \cdot u_{D} ">0$ and $H<W_{L}-W_{D}$ i.e. expected wealth decreases with an increase in survival probability, or $u_{L} " \cdot u_{D} "<0$ and $H>W_{L}-W_{D}$ i.e. a positive income effect. In the opposite constellations, the DAE fails.

In the standard case with concave utility in both states of nature, $H=0$ and $W_{L}>W_{D}$ guarantee the DAE to hold. With $H>0$, the sign of $\partial m_{b} / \partial \pi$ is indeterminate. Provided that at the initial equilibrium $\left(W_{L}, W_{D}\right)$ an increase in the chance of survival enlarges the opportunity set, the individual will want to extend her demand for annuities. We conclude that even in the standard case, the DAE does not necessarily carry over to the bequest model if the individual is endowed with non-inheritable wealth.

With respect to the inheritable wealth derivative we obtain:

$$
\frac{\partial m_{b}(\pi, V, H)}{\partial V}=-\frac{\left(u_{L}-u_{D}\right) \cdot u_{L}{ }^{\prime}}{\left(u_{L}^{\prime}\right)^{2}} \cdot \frac{\partial W_{L}}{\partial V} .
$$

As demand for the annuity increases with initial inheritable wealth in the standard case, noninferiority of survival prevails in the bequest model. However, for the general case we find (see the Appendix):

$$
\frac{\partial m_{b}(\pi, V, H)}{\partial V}=-\frac{\left(u_{L}-u_{D}\right)}{\left(u_{L}^{\prime}\right)^{2}} \cdot \frac{u_{L} " \cdot u_{D} "}{a},
$$

and $u_{L}{ }^{\prime \prime} \cdot u_{D} "<0$ gives the opposite sign.

For the effect of a change in non-inheritable wealth on the value of a statistical life, we find:

$$
\frac{\partial m_{b}(\pi, V, H)}{\partial H}=1-\left(u_{L}-u_{D}\right) \cdot \frac{\pi \cdot u_{L} "}{\left(u_{L}^{\prime}\right)^{2}} .
$$

Thus again, concavity of the utility function is a sufficient but not a necessary condition for the value of life to increase with the level of non-inheritable wealth. However, steeply increasing marginal utility for the state of survival $\left(u_{L} ">0\right)$ at the given wealth might reverse the effect of a change in non-inheritable wealth on the WTP for survival.

\section{Imperfect insurance markets}

If mortality risk cannot be insured, the budget constraint in the no-bequest model changes to: 


$$
W=V+H,
$$

so that the distinction between inheritable and non-inheritable wealth can be dropped and the marginal rate of substitution between survival and wealth becomes: ${ }^{2}$

$$
m_{n i}(\pi, V+H)=\frac{u}{\pi \cdot u^{\prime}}
$$

giving rise to:

$$
\frac{\partial m_{n i}(\pi, V+H)}{\partial \pi}=-\frac{u}{\pi^{2} \cdot u^{\prime}}<0
$$

The DAE is still present, but this time not caused by the concavity of the utility function. Marginal utility is not affected by the change in mortality risk as the consumption level in the state of survival remains constant. This effect comes through the objective function: expected marginal utility of consumption falls when mortality risk rises. This is the DAE that Pratt and Zeckhauser had in mind.

The derivative with respect to initial wealth yields:

$$
\frac{\partial m_{n i}(\pi, V+H)}{\partial(V+H)}=\frac{1}{\pi}\left[1-\frac{u \cdot u^{\prime \prime}}{\left(u^{\prime}\right)^{2}}\right] .
$$

Here, we have a similar result to non-inheritable wealth in the perfect market case: risk aversion is a sufficient but not a necessary condition for the WTP for survival to increase with initial wealth.

\section{Combining a bequest motive with imperfect insurance markets}

In the bequest model with non-existent insurance markets, the budget constraints in the two states are:

$$
W_{L}=V+H \text { and } W_{D}=V \text {. }
$$

Marginal willingness to pay for survival is then:

$$
m_{b, n i}(\pi, V, H)=\frac{u_{L}(V+H)-u_{D}(V)}{E U^{\prime}}
$$

with $E U^{\prime}=\pi \cdot u_{L}{ }^{\prime}+(1-\pi) \cdot u_{D}{ }^{\prime}$ as the expected marginal utility of wealth. For the derivative with respect to $\pi$ we obtain: 


$$
\frac{\partial m_{b, n i}(\pi, V, H)}{\partial \pi}=-\left(u_{L}-u_{D}\right) \cdot \frac{u_{L}{ }^{\prime}(V+H)-u_{D}{ }^{\prime}(V)}{\left(E U^{\prime}\right)^{2}} .
$$

Interestingly, the RHS of (26) can not be signed because the sign of the numerator is indeterminate for positive values of non-inheritable wealth. The intuition for the case of a positive sign of $\partial m_{n i, b} / \partial \pi$ is the following: If

$$
u_{L}{ }^{\prime}(V+H)<u_{D}{ }^{\prime}(V) \text {, }
$$

the marginal utility of wealth upon death exceeds the one upon survival because noninheritable wealth is lost and inheritable wealth can not be shifted to the heirs due to the absence of a life insurance market. An increase in the risk of death, therefore raises the expected marginal utility of wealth and thus decreases marginal WTP for survival - the opposite of the DAE.

No surprises arise with respect to the wealth elasticity of the value of a statistical life. Once more, risk aversion is only a sufficient condition to create the expected sign. In fact, a derivation of (25) with respect to inheritable wealth gives:

$$
\frac{\partial m_{b, n i}(\pi, V, H)}{\partial V}=\frac{u_{L}{ }^{\prime}-u_{D}{ }^{\prime}}{E U^{\prime}}-\left(u_{L}-u_{D}\right) \cdot \frac{\pi \cdot u_{L}{ }^{\prime \prime}+(1-\pi) \cdot u_{D} "}{\left(E U^{\prime}\right)^{2}}>0
$$

Regarding the effect of a change in non-inheritable health we find:

$$
\frac{\partial m_{b, n i}(V, H, \pi)}{\partial H}=\frac{u_{L}{ }^{\prime}}{E U^{\prime}}-\left(u_{L}-u_{D}\right) \cdot \frac{\pi \cdot u_{L}{ }^{\prime \prime}}{\left(E U^{\prime}\right)^{2}}
$$

As life insurance policies can not be written, the heirs do not benefit from a change in noninheritable wealth. Hence, marginal WTP for survival exclusively depends on the utility function in the state of survival, except for the utility difference between the two states of nature. Again, concavity of $u_{L}$ is a sufficient condition for WTP to increase with noninheritable wealth.

\section{Conclusion}

In this study, we have reexamined the claim made by Pratt and Zeckhauser (1996) of a "deadanyway" effect on the value of a statistical life, stating that WTP for survival increases with the level of initial mortality risk. We have analyzed this hypothesis in models with and

\footnotetext{
${ }^{2}$ The subscript $n i$ at the marginal WTP refers to the case of no-insurance.
} 
without bequests and under perfect and imperfect insurance markets. The insights which emerge from the theoretical analysis are as follows:

First, for individuals without a bequest motive the value of a statistical life always increases with the level of risk exposure if and only if they are risk-averse with respect to wealth. However, with perfect contingent-claims markets, the label "dead-anyway effect" does not corresponds with our theoretical result; since in this case the driving force is not the reduced marginal utility of wealth in the state of death, but the increase in the size of the annuity.

Secondly, when a bequest motive exists, a reversal of the effect of risk level on WTP for survival may occur - again, independent of the existence of perfect insurance markets. A sufficient condition for this to happen is that a significant portion of total wealth is held as non-inheritable capital, which of course is lost when the individual dies. Consequently, the necessary correction of survey results for differences in the level of risk exposure may have the opposite direction compared to Pratt and Zeckhauser.

Thirdly, WTP for survival depends on the individual's inheritable and non-inheritable wealth in different ways: whereas for inheritable wealth risk aversion is a necessary and sufficient condition for a positive sign, for non-inheritable wealth concavity of the utility function is sufficient but not necessary. We would like to mention that all results regarding the effects of non-inheritable wealth remain valid if labor supply is endogenous. This is an immediate implication of the envelope theorem.

The theoretical examination performed in this paper can help to explain why the empirical evidence on the relation between WTP and the level of risk exposure is so inconclusive. ${ }^{3}$ For instance, Smith and Desvousges (1987) found in their questionnaire study that "in the majority of cases ... the bids are larger at smaller baseline risks" (ibid., p. 99). Similarly, Breyer and Grabka (2001) analyzing data from a large panel study, find no significant effect of risk measures such as age and recent hospitalizations on the marginal utility of wealth, thus indirectly casting doubt on the presence of a "dead-anyway" effect.

A negative "dead-anyway" effect may be an explanation for the fact that markets for private annuities are more or less inexistent in the industrialized world. It is well known that private markets for annuities are crowded out by extensive pay-as-you-go and funded social old-age

\footnotetext{
${ }^{3}$ For extensive reviews of the empirical literature, see Viscusi (1993) and more recently, Hammitt and Graham (1999).
} 
insurance. These schemes, however, increase non-inheritable wealth, providing a further rationale for the individuals' reluctance to buy annuities.

\section{References}

Breyer, F. and M. Grabka (2001), Is there a dead-anyway effect in willingness to pay for risk reduction?, Discussion Paper No. 252, DIW Berlin, http://www.diw.de/english/publikationen/diskussionspapiere/jahrgang01/.

Felder, S., M. Meier and H. Schmitt (2000), Health Care Expenditure in the Last Months of Life, Journal of Health Economics 19(5), 679-695.

Johannson, P.-O. (1996), On the value of a statistical life, Journal of Health Economics 15, 105-113.

Hammitt, J.K. and J.D. Graham (1999), Willingness to Pay for Health Protection: Inadequate Sensitivity to Probability?, Journal of Risk and Uncertainty 18, 33-62.

Jones-Lee, M.-W. (1976), The Value of Life: An Economic Analysis, Chicago: University Press.

Pratt, J. W. and R. J. Zeckhauser (1996), Willingness to Pay and the Distribution of Risk and Wealth, Journal of Political Economy 104, 747-763.

Rosen, S. (1988), The Value of Changes in Life Expectancy, Journal of Risk and Uncertainty $1,285-304$.

Smith, V.K. and W.H. Desvousges (1987), An Empirical Analysis of the Economic Value of Risk Changes, Journal of Political Economy 95, 89-114.

Viscusi, W. K. (1993), The Value of Risks to Life and Health, Journal of Economic Literature 31, 1912-1946. 


\section{Appendix: Properties of the bequest model with perfect insurance markets}

\section{Second-order condition for the (constrained) maximum}

The second-order condition is satisfied if the indifference curves are convex. For the indifference curve we obtain:

$$
\left.\frac{d W_{D}}{d W_{L}}\right|_{d E U=0}=-\frac{\pi}{1-\pi} \cdot \frac{u_{L}{ }^{\prime}\left(W_{L}\right)}{u_{D}{ }^{\prime}\left(W_{D}\right)} .
$$

In order to derive the curvature of the indifference curve, we form the second derivative:

$$
\left.\frac{d^{2} W_{D}}{\left(d W_{L}\right)^{2}}\right|_{d E U=0}=-\frac{\pi}{1-\pi} \cdot \frac{u_{L} " u_{D}{ }^{\prime}-u_{D}{ }^{\prime \prime} \cdot d W_{D} / d W_{L} \cdot u_{L}{ }^{\prime}}{\left(u_{D}{ }^{\prime}\right)^{2}} .
$$

Since $d W_{D} / d W_{L}=-\pi /(1-\pi)$ and using the FOC (11), we find:

$$
\frac{d^{2} W_{D}}{\left(d W_{L}\right)^{2}}>0 \text {, if and only if } a:=\pi \cdot u_{D} "+(1-\pi) \cdot u_{L} "<0 \text {. }
$$

Three cases satisfy the condition $a<0$ :

i) $\quad u_{D} "<0, \quad u_{L} "<0$

ii) $\quad u_{D}{ }^{\prime \prime}>0, \quad u_{L}{ }^{\prime \prime}<0$ and $\pi \cdot\left|u_{D} "\right|<(1-\pi) \cdot\left|u_{L} "\right|$

iii) $\quad u_{D}{ }^{\prime \prime}<0, \quad u_{L}{ }^{\prime}>0$ and $\pi \cdot\left|u_{D} "\right|>(1-\pi) \cdot\left|u_{L} "\right|$

2. Equation (15): $\partial W_{L} / \partial \pi$

From the budget constraint (10), we find:

$$
\begin{aligned}
& W_{L}+\pi \cdot \frac{\partial W_{L}}{\partial \pi}-W_{D}+(1-\pi) \cdot \frac{\partial W_{D}}{\partial \pi}=H \text { or } \\
& \pi \cdot \frac{\partial W_{L}}{\partial \pi}+(1-\pi) \cdot \frac{\partial W_{D}}{\partial \pi}=H-\left(W_{L}-W_{D}\right),
\end{aligned}
$$

Total differentiation of the FOC (11) yields:

$$
u_{L} " \cdot \frac{\partial W_{L}}{\partial \pi}=u_{D} " \cdot \frac{\partial W_{D}}{\partial \pi} \quad \text { or } \quad \frac{\partial W_{D}}{\partial \pi}=\frac{u_{L} "}{u_{D} "} \cdot \frac{\partial W_{L}}{\partial \pi} .
$$

Inserting into (33) gives:

$$
\begin{aligned}
& \frac{\partial W_{L}}{\partial \pi} \cdot\left[\pi+(1-\pi) \cdot \frac{u_{L} "}{u_{D} "}\right]=H-\left(W_{L}-W_{D}\right) \text {, or } \\
& \frac{\partial W_{L}}{\partial \pi}=\left[H-\left(W_{L}-W_{D}\right)\right] \cdot \frac{u_{D} "}{a}, \text { which corresponds to (15) in the text. }
\end{aligned}
$$


3. Equation (18): $\partial W_{L} / \partial V$

Again from (10) we have:

$$
\pi \cdot \frac{\partial W_{L}}{\partial V}+(1-\pi) \cdot \frac{\partial W_{D}}{\partial V}=1 .
$$

The FOC (11) provides an expression for $\partial W_{D} / \partial V$ (analogous to (34)) and we arrive at:

$$
\frac{\partial W_{L}}{\partial V}=\frac{u_{D} "}{a} .
$$

(18) then immediately follows from (17). 